\title{
RADIOCARBON DATES FOR TASMANIA, 1956-1984
}

\author{
by Eric A. Colhoun
}

COLHOUN, E.A., 1985 (31:vii): Radiocarbon dates for Tasma nia, 1956-1984. Pap. Proc. R. Soc. Tasm. 119:39-54. https://doi.org/10.26749/rstpp.119.39 ISSN 0080-4703. Department of Geography, University of Tasmania. GPO Box 252C, Hobart, 7001, Tasmania. Australia.

A list of radiocarbon dates from Tasmania and Macquarie Island is provided.

Key Words:- radiocarbon dates, Tasmania, Macquarie Island, geomorphology, palynology.

\section{INTRODUCTION}

Several hundred ${ }^{14} \mathrm{C}$ dates have been obtained from Tasmania, including Macquarie Island, during geomorphological and palynological studies of the Quaternary in the past three decades. While many dates have been published in the studies concerned others have not, and their existence is not generally known. The appended tables contain all known ${ }^{14} \mathrm{C}$ dates to December 1984, except for dates mainly of archaeological significance which have been compiled by Stockton until 1981 .

The locality of the site is shown to the nearest hundred metres by providing a grid reference (GR) consisting of the number (e.g. 31) of the appropriate 1:100 000 sheet (third series) of the Department of Lands, Tasmania, together with six figures indicating eastings and northings. The abbreviation of the sheet number can be restored to the full number by inserting " 7 " before the first digit of the abbreviation if that digit is 6 or more, " 8 " if that digit is 5 or less, and by inserting the number "I" between the first and second digits of the abbreviation. Thus " 31 " is expanded to " 8311 ", "94" to "7914".
All ${ }^{14} \mathrm{C}$ ages a re given using the Libby half-life of 5568 years. Isotope-enriched assays of Groningen University $(\mathrm{GrN})$ are given in brackets and were made using the method of Grootes (1978). An index to labora tory codes immediately follows this introduction.

The inclusion of previously unpublished dates has been made possible by the willingness of the research workers concerned, whose names are noted in brackets in the reference column and whose addresses are listed after the tabulated section.

The purpose of listing the dates is to (1) make their existence known, and (2) to commence a ${ }^{14} \mathrm{C}$ date bank for Tasmania. To allow maintenance of the date bank workers who obtain ${ }^{14} \mathrm{C}$ dates for Tasmania are asked to forward their results to the author in the same form as produced in this listing, for computer storage until a later list is published. Some extant dates may have been inadvertently omitted from the present list. If so, details of the dates concerned would be gratefully received by the author.

\section{LABORATORY CODES}

ANU Australian National University, Canberra, Australia

ARL Australian Radiometric Laboratories, Sydney, Australia

Beta Beta Analytic, Coral Gables, Florida, U.S.A.

Gak Gakushuin University, Tokyo, Japan

GrN University of Groningen, Netherlands

GX Geochron Laboratories, Cambridge, Mass., U.S.A.

I Teledyne Isotopes, Westwood, New Jersey, U.S.A.

NSW University of New South Wales, Kensington, Australia
NZ New Zealand, Institute of Nuclear Sciences, Lower Hutt, N.Z.

Pta CSIR, Pretoria, South Africa

R University of Rome, Italy

SUA Sydney University, Australia

UB Queens University of Belfast, Northern Ireland

$\mathrm{V}$ Institute of Applied Science, Melbourne, Victoria, Australia

Y Yale University, New Haven, Conn., U.S.A.

W U.S. Geological Survey, Reston, Virginia, U.S.A.

WK University of Waikato, New Zealand 


\section{TABULATION OF INFORMATION ON RADIOCARBON DATES}

\section{TASMANIA}

\section{Modern Materials}

\begin{tabular}{|c|c|c|c|c|c|c|c|}
\hline Site Name & GR Ref & Material & ${ }^{14} \mathrm{C}$ & S D & Lab No & Reference & Comment \\
\hline \multirow[t]{3}{*}{ Adventure Bay } & $31-294985$ & $\begin{array}{l}\text { shell of } \\
\text { Cellana } \\
\text { solida }\end{array}$ & 114.6 & $0.9 \%$ & SUA $294 / 2$ & Gillespie 1977 & $\begin{array}{l}\text { nuclear test effects in } \\
\text { southwestern Pacific }\end{array}$ \\
\hline & & $\begin{array}{l}\text { meat of } \\
C . \text { solida }\end{array}$ & 117.2 & $1.1 \%$ & SUA $294 / 2 M$ & & \\
\hline & & $\begin{array}{l}\text { shell of } \\
\text { Austro- } \\
\text { cochlea } \\
\text { odontis }\end{array}$ & 112.9 & $1.2 \%$ & SUA $294 / 3$ & & \\
\hline Eaglehawk Neck & $41-759377$ & $\begin{array}{l}\text { shell of } \\
\text { Brachio- } \\
\text { dontes } \\
\text { rostratis }\end{array}$ & 110.9 & $0.9 \%$ & SUA $294 / 1$ & Gillespie 1977 & nuclear effects \\
\hline \multirow[t]{9}{*}{ Lake Gordon } & $\begin{array}{l}\text { 12- } \\
\text { tree cut } \\
\text { by IXL, } \\
\text { location } \\
\text { not known }\end{array}$ & $\begin{array}{l}1941-45 \\
\text { wood of } \\
\text { Lagaros- } \\
\text { trobos } \\
\text { franklinii }\end{array}$ & & & & & \\
\hline & & & & $\delta^{3} C$ & $\Delta^{14} C$ & & \\
\hline & & $\begin{array}{l}\text { benzene- } \\
\text { ethanol } \\
\text { extract }\end{array}$ & -24.8 & $318 \pm 18$ & SUA 5002 & $\begin{array}{l}\text { Francey et al. } \\
1984\end{array}$ & $\begin{array}{l}\text { pretreatment using } \\
\text { solvent/acid/alkali/ } \\
\text { acid method }\end{array}$ \\
\hline & & $\begin{array}{l}\text { acid } \\
\text { extract }\end{array}$ & -20.1 & $-2 . \pm 9$ & SUA 5006 & & \\
\hline & & $\begin{array}{l}\text { alkali } \\
\text { extract }\end{array}$ & -25.1 & $107 \pm 37$ & SUA 5007 & & \\
\hline & & $\begin{array}{l}\text { wood } \\
\text { residue }\end{array}$ & -22.9 & $-16 \pm 6$ & SUA 5005 & & \\
\hline & & $\begin{array}{l}\text { acid } \\
\text { extract }\end{array}$ & -20.1 & $21 \pm 11$ & SUA 5001 & & $\begin{array}{l}\text { pretreatment using } \\
\text { acid/alkali/acid } \\
\text { method }\end{array}$ \\
\hline & & $\begin{array}{l}\text { alkali } \\
\text { extract }\end{array}$ & -25.0 & $226 \pm 21$ & SUA 5003 & & \\
\hline & & $\begin{array}{l}\text { wood } \\
\text { residue }\end{array}$ & -22.6 & $-5 \pm 6$ & SUA 5000 & & \\
\hline \multirow[t]{2}{*}{ Key Island } & $57-870232$ & $\begin{array}{l}\text { shells of } \\
\text { Subninella } \\
\text { undulata }\end{array}$ & 455 & 120 & GX 7009 & (Gill) & $\begin{array}{l}\text { coll. } 2-1-1956 \text { at } \\
\text { spring L.W. Pre A- } \\
\text { bomb testing } \\
\text { in Pacific }\end{array}$ \\
\hline & & & & $\delta^{13} \mathrm{C}$ & $\Delta^{14} C$ & & \\
\hline \multirow[t]{2}{*}{ Stanley River } & $94-565780$ & leaf & -29.6 & $286 \pm 9$ & SUA 5008 & $\begin{array}{l}\text { McPhail et al. } \\
\text { I983 }\end{array}$ & $\begin{array}{l}\text { leaves of } \\
\text { Phyllocladus asplenit- } \\
\text { folius from below } \\
\text { and above canopy }\end{array}$ \\
\hline & & leaf & -24.0 & $293 \pm 7$ & SUA 5009 & $\begin{array}{l}\text { Francey et al. } \\
1984\end{array}$ & \\
\hline
\end{tabular}




\section{Aeolian Landforms and Deposits}

\begin{tabular}{|c|c|c|c|c|c|c|c|}
\hline Site Name & GR Ref & Material & ${ }^{14} \mathrm{C}$ & S D & Lab No & Reference & Comment \\
\hline Bridgewater & $32-167682$ & charcoal & 4540 & 105 & Gak 5593 & $\begin{array}{l}\text { Sigleo \& } \\
\text { Colhoun } 1982\end{array}$ & midden base on dune \\
\hline \multirow[t]{3}{*}{ Glenfield } & $32-215696$ & $\begin{array}{l}\text { charcoal } \\
\text { carb. wood }\end{array}$ & 210 & 80 & SUA 303 & $\begin{array}{l}\text { Sigleo \& } \\
\text { Colhoun } 1982\end{array}$ & hearths in dune \\
\hline & & charcoal & 1245 & 80 & SUA 304 & & \\
\hline & & charcoal & 2055 & 80 & SUA 305 & & \\
\hline $\begin{array}{l}\text { Malcolms Hut } \\
\text { Road }\end{array}$ & $32-335643$ & charcoal & 15740 & 700 & SUA 376 & Colhoun 1975 & Pleistocene dune \\
\hline \multirow[t]{4}{*}{$\begin{array}{l}\text { Pipe Clay } \\
\text { Lagoon }\end{array}$} & $42-433428$ & $\begin{array}{l}\text { org. sand } \\
\text { and clay }\end{array}$ & 19810 & 360 & SUA 153 & Colhoun $1977 \mathrm{a}$ & $\begin{array}{l}\text { Pleistocene dune- } \\
\text { filled wet depression }\end{array}$ \\
\hline & & $\begin{array}{l}\text { org. sand } \\
\text { and clay }\end{array}$ & 21905 & 440 & SUA $153 / 2$ & & \\
\hline & & org. clay & 20250 & 360 & SUA 152 & & \\
\hline & & org. clay & 25380 & 640 & SUA 151 & & \\
\hline South Arm Beach & $31-370352$ & charcoal & 490 & 110 & Gak 6295 & (Colhoun) & $\begin{array}{l}\text { midden with dune } \\
\text { above and below }\end{array}$ \\
\hline \multirow[t]{3}{*}{ Rushy Lagoon } & $56-868758$ & charcoal & 8300 & 80 & Beta 8190 & Cosgrove 1985 & $\begin{array}{l}\text { hearth } 0.4-0.7 \mathrm{~m} \\
\text { depth in lunette }\end{array}$ \\
\hline & & wood & 8570 & 135 & $1-11448 \mathrm{~A}$ & $\begin{array}{l}\text { Sigleo \& } \\
\text { Colhoun } 1982\end{array}$ & $\begin{array}{l}\text { organic beds below } \\
\text { Holocene lunette }\end{array}$ \\
\hline & & hum. acid & 8435 & 185 & $1-11448$ B & & \\
\hline
\end{tabular}

\section{Cave Landforms and Deposits}

\begin{tabular}{|c|c|c|c|c|c|c|c|}
\hline Site Name & GR Ref & Material & ${ }^{14} \mathrm{C}$ & S D & Lab No & Reference & Comment \\
\hline Baldocks Cave & $14-452966$ & charcoal & 12500 & $\begin{array}{l}+3300 \\
-2400\end{array}$ & ARL 222 & (Kiernan) & base of silt on gravel \\
\hline Bone Cave & $12-557570$ & charcoal & 16200 & $\begin{array}{l}+1200 \\
-1100\end{array}$ & SUA 2104 & (Goede) & archaeological site \\
\hline \multirow{2}{*}{$\begin{array}{l}\text { Breccia Ridge } \\
\text { Cave }\end{array}$} & $12-554872$ & charcoal & 7380 & 130 & Gak 7036 & (Goede) & cave entrance deposit \\
\hline & & charcoal & 8290 & 280 & Gak 7034 & & \\
\hline Flowery Gully & $25-850327$ & $\begin{array}{l}\text { charcoal \& } \\
\text { bone }\end{array}$ & 7080 & 420 & Gak 967 & Gill 1968 & $\begin{array}{l}\text { bone bed in cave } \\
\text { earth }\end{array}$ \\
\hline \multirow[t]{6}{*}{ Frankcombe Cave } & $12-550910$ & calcite & 3630 & 65 & Pta 2505 & (Goede) & stalagmite \\
\hline & & calcite & 5490 & 70 & Pta 2575 & & \\
\hline & & calcite & 5790 & 70 & Pta 2574 & & \\
\hline & & calcite & 5990 & 70 & Pta 2571 & & \\
\hline & & calcite & 6320 & 70 & Pta 2499 & & \\
\hline & & calcite & $>41000$ & & SUA 2208 & & \\
\hline \multirow[t]{9}{*}{ Lynds Cave } & 14-357974 & calcite & 5250 & 70 & Pta 2979 & $\begin{array}{l}\text { Goede \& } \\
\text { Hitchman } 1984\end{array}$ & stalagmite \\
\hline & & calcite & 6400 & 20 & Pta 3199 & & \\
\hline & & calcite & 8980 & 90 & Pta 2976 & & \\
\hline & & calcite & 10300 & 90 & Pta 2975 & & \\
\hline & & calcite & 13000 & 30 & Pta 3198 & & \\
\hline & & calcite & 14200 & 90 & Pta 3713 & & \\
\hline & & calcite & 14500 & 140 & $\operatorname{Pta} 2972$ & & \\
\hline & & calcite & 13400 & 130 & Pta 3708 & & \\
\hline & & calcite & 12700 & 90 & Pta 3707 & & \\
\hline
\end{tabular}


Cave Landforms and Deposits

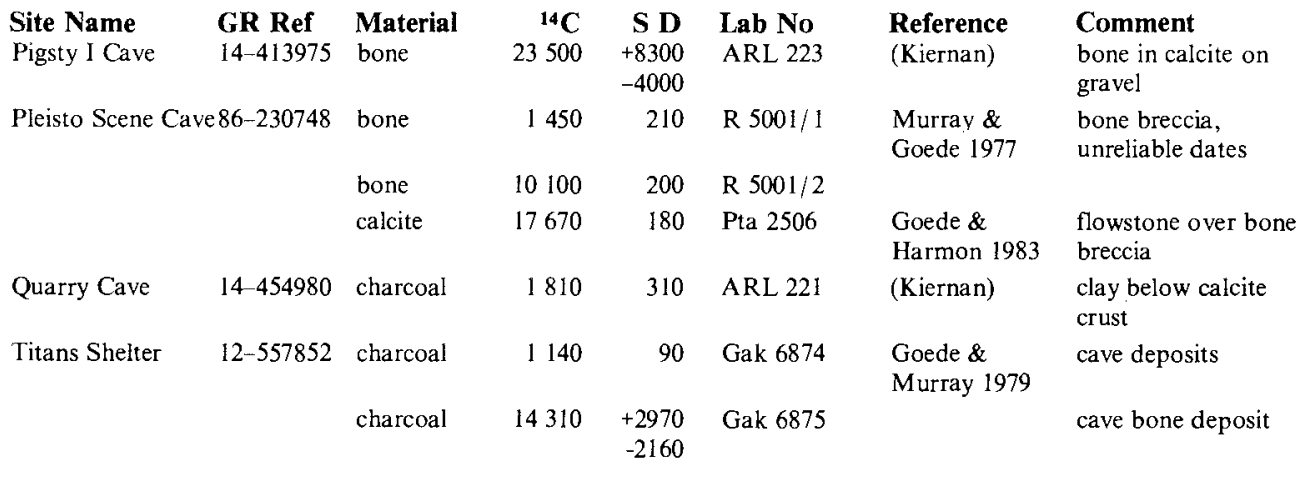

\section{Coastal Landforms and Marine Sediments}

\begin{tabular}{|c|c|c|c|c|c|c|c|}
\hline Site Name & GR Ref & Material & ${ }^{14} \mathrm{C}$ & S D & Lab No & Reference & Comment \\
\hline \multirow[t]{3}{*}{ Anthonys Beach } & $96-471805$ & shell hash & 3500 & 60 & SUA 1301 & $\begin{array}{l}\text { Thom et al. } \\
1981\end{array}$ & barrier transect \\
\hline & & shell hash & 1780 & 55 & SUA 1302 & & \\
\hline & & shell hash & 7660 & 75 & SUA 1303 & & \\
\hline \multirow[t]{15}{*}{ Bowen Bridge } & $32-250593$ & org. mud & 790 & 70 & SUA 1960 & $\begin{array}{l}\text { Colhoun \& } \\
\text { Moon } 1984\end{array}$ & $\begin{array}{l}-10.5 \mathrm{~m} \text { drill hole } 2 \\
\text { piers } 2 \& 3\end{array}$ \\
\hline & & shell & 1490 & 70 & SUA 1961 & & $-16.1 \mathrm{~m}$ \\
\hline & & org. mud & 3080 & 80 & SUA 1962 & & $-19.0 \mathrm{~m}$ \\
\hline & & shell & 2060 & 150 & SUA 1963 & & $-26.3 m$ \\
\hline & & shell & 4440 & 150 & SUA 1964 & & $-29.5 m$ \\
\hline & & org. mud & 7370 & 140 & SUA 1965 & & $-29.5 m$ \\
\hline & & org. mud & 4490 & 90 & SUA 1966 & & $-30.0 \mathrm{~m}$ \\
\hline & & org. mud & 9070 & 110 & SUA 1967 & & $-35.5 m$ \\
\hline & & shell & 9150 & 290 & SUA 1968 & & $-38.9 m$ \\
\hline & & shell & 9060 & 300 & SUA 1969 & & $-40.0 \mathrm{~m}$ \\
\hline & & org. mud & 9390 & 110 & SUA 1970 & & $-42.5 \mathrm{~m}$ \\
\hline & & shell & 2610 & 100 & SUA 1971 & & $-24.0 \mathrm{~m}$ pier 6 \\
\hline & & shell & 2560 & 160 & SUA 1972 & & $-32.4 \mathrm{~m}$ pier 4 \\
\hline & & shell & 3190 & 180 & SUA 1973 & & $\begin{array}{c}-10.3 \text { to }-10.5 \mathrm{~m} \\
\text { drill hole } 10\end{array}$ \\
\hline & & shell & 7960 & 320 & SUA 1974 & & $\begin{array}{l}-42.9 \text { to }-43.2 \mathrm{~m} \\
\text { drill hole } 2\end{array}$ \\
\hline Cremorne & $42-429439$ & carb. wood & 3620 & 80 & Gak 650 & $\begin{array}{l}\text { Kigoshi \& } \\
\text { Kobayashi } 1966\end{array}$ & $\begin{array}{l}\text { beach sands } 1.3 \mathrm{~m} \\
\text { above HWMST }\end{array}$ \\
\hline \multirow[t]{4}{*}{ Greens Beach } & $25-787515$ & shell hash & 6085 & 90 & SUA 1297 & $\begin{array}{l}\text { Thom et al. } \\
1981\end{array}$ & barrier transect \\
\hline & & shell hash & 38765 & $\begin{array}{l}+2700 \\
-2015\end{array}$ & SUA 1298 & & \\
\hline & & shell hash & 8005 & 90 & SUA 1299 & & \\
\hline & & shell hash & 3770 & 70 & SUA 1300 & & \\
\hline Laycocks Beach & $15-455429$ & $\begin{array}{l}\text { charcoal \& } \\
\text { carb. wood }\end{array}$ & 5990 & 260 & Gak 5618 & Colhoun 1983a & $\begin{array}{l}\text { present sea level } \\
\text { already attained }\end{array}$ \\
\hline Marion Bay & $42-710572$ & carb. wood & 390 & 90 & Gak 647 & $\begin{array}{l}\text { Kigoshi \& } \\
\text { Kobayashi } 1966\end{array}$ & $\begin{array}{l}\text { sample } 0.2 \mathrm{~m} \text { asl in } \\
\text { barrier beach }\end{array}$ \\
\hline $\begin{array}{l}\text { Nelsons Drain, } \\
\text { Flinders Island }\end{array}$ & $57-0 \mathrm{I} 0 \ldots$ & shell & 21620 & 750 & Gak 8256 & (Ladd) & shell from drain wall \\
\hline
\end{tabular}




\begin{tabular}{|c|c|c|c|c|c|c|c|}
\hline \multirow{3}{*}{$\begin{array}{l}\text { Site Name } \\
\text { Nilinga Creek, } \\
\text { Flinders Island }\end{array}$} & \multicolumn{6}{|c|}{ Coastal Landforms and Marine Sediments } & \multirow{3}{*}{$\begin{array}{l}\text { Comment } \\
\text { beach gravel }+1.8 \mathrm{~m}\end{array}$} \\
\hline & GR Ref & Material & ${ }^{14} \mathrm{C}$ & S D & Lab No & Reference & \\
\hline & $57-865565$ & shell & 3990 & 100 & SUA 413 & (Gill) & \\
\hline \multirow[t]{6}{*}{ Nine Mile Beach } & $53-905395$ & shell hash & 5760 & 80 & SUA 1291 & $\begin{array}{l}\text { Thom et al. } \\
\text { 1981 }\end{array}$ & barrier transect \\
\hline & & shell hash & 9465 & 105 & SUA 1292 & & \\
\hline & & shell hash & 11350 & 125 & SUA 1293 & & \\
\hline & & shell hash & 8770 & 120 & SUA 1294 & & \\
\hline & & shell hash & 11110 & 175 & SUA 1295 & & \\
\hline & & shell hash & 8575 & 105 & SUA 1296 & & \\
\hline \multirow[t]{8}{*}{ Rheban } & $42-777777$ & shell hash & 3445 & 140 & GX 4021 & $\begin{array}{l}\text { Thom et al. } \\
1981\end{array}$ & barrier transect \\
\hline & & shell hash & 3550 & 150 & GX 4020 & & \\
\hline & & shell hash & 4330 & 125 & SUA 768/1 & & \\
\hline & & wood & 815 & 125 & SUA $768 / 2$ & & \\
\hline & & shell hash & 4750 & 180 & GX 4363 & & \\
\hline & & shell hash & 5310 & 150 & GX 4022 & & \\
\hline & & shell hash & 4330 & 170 & GX 4364 & & \\
\hline & & shell hash & 4340 & 120 & GX 4365 & & \\
\hline \multirow[t]{3}{*}{ Rocky Cape } & $06-743753$ & shell & $<190$ & - & unknown & (Stephens) & $\begin{array}{l}\text { beach } 1.8 \mathrm{~m} \text { above } \\
\text { HWMST east side of } \\
\text { Rocky Cape }\end{array}$ \\
\hline & $06-\ldots \ldots$ & shell & 3795 & 100 & V 83 & & $\begin{array}{l}\text { probably west side of } \\
\text { Rocky Cape }\end{array}$ \\
\hline & $06-\ldots \ldots$ & shell & 3434 & 95 & V 88 & & \\
\hline Seven Mile Beach & $42-470581$ & shell & 4140 & 90 & SUA 1486 & $\begin{array}{l}\text { Thom et al. } \\
1981\end{array}$ & $\begin{array}{l}\text { near base of deposit } \\
\text { below swale south of } \\
\text { inner beach ridge }\end{array}$ \\
\hline \multirow[t]{2}{*}{ Snug } & $31-211321$ & shell & 510 & 80 & Gak 1143 & $\begin{array}{l}\text { Kigoshi \& } \\
\text { Kobayashi } 1966\end{array}$ & $\begin{array}{l}\text { sand } 0.7 \mathrm{~m} \text { below } \\
\text { HWMST }\end{array}$ \\
\hline & & shell & 2760 & 120 & Gak 649 & & $\begin{array}{l}\text { sand } 1.7 \mathrm{~m} \text { below } \\
\text { HWMST }\end{array}$ \\
\hline $\begin{array}{l}\text { The Jam, } \\
\text { Smithton }\end{array}$ & $96-353830$ & shell & 22700 & 1100 & Gak 652 & $\begin{array}{l}\text { Kigoshi \& } \\
\text { Kobayashi } 1966\end{array}$ & $\begin{array}{l}\text { derived Pleistocene } \\
\text { shell at }-3 m\end{array}$ \\
\hline Tinderbox & $31-270325$ & shell & 260 & 70 & Gak 648 & $\begin{array}{l}\text { Kigoshi \& } \\
\text { Kobayashi } 1966\end{array}$ & $\begin{array}{l}\text { beach } 0.5-2 \mathrm{~m} \text { above } \\
\text { HWMST }\end{array}$ \\
\hline $\begin{array}{l}\text { Yellow Beaches, } \\
\text { Flinders Island }\end{array}$ & $57-064479$ & wood & 3970 & 90 & Gak 1102 & (Gill) & $\begin{array}{l}\text { at Lady Barron } \\
\text { below Henwoods } \\
\text { House }\end{array}$ \\
\hline
\end{tabular}

\section{Fluvial Landforms and Deposits}

\begin{tabular}{|c|c|c|c|c|c|c|c|}
\hline Site Name & GR Ref & Material & ${ }^{14} \mathrm{C}$ & S D & Lab No & Reference & Comment \\
\hline \multirow[t]{4}{*}{ Barilla Valley } & $32-378583$ & charcoal & 550 & 80 & Gak? & (Stephens) & alluvial gravel \\
\hline & $32-353572$ & charcoal & 1160 & 100 & Gak 651 & $\begin{array}{l}\text { Kigoshi \& } \\
\text { Kobayashi } 1966\end{array}$ & alluvial gravel \\
\hline & $32-343564$ & charcoal & 4650 & 120 & Gak 488 & & alluvial gravel \\
\hline & & charcoal & 7900 & 460 & Gak 487 & & alluvial gravel \\
\hline \multirow[t]{4}{*}{ Blakes Opening } & $21-690279$ & charcoal & $>29150$ & & Gak 5587 & $\begin{array}{l}\text { Colhoun \& } \\
\text { Goede } 1979\end{array}$ & $\begin{array}{l}\text { alluvium containing } \\
\text { organic beds }\end{array}$ \\
\hline & & charcoal & 27400 & 2900 & Gak 5588 & & \\
\hline & & charcoal & 29340 & $\begin{array}{r}+3080 \\
-2220\end{array}$ & Gak 5589 & & \\
\hline & & charcoal & 39600 & 1000 & GrN 7695 & & \\
\hline
\end{tabular}


Fluvial Landforms and Deposits

\begin{tabular}{|c|c|c|c|c|c|c|c|}
\hline \multirow[t]{3}{*}{ Site Name } & \multirow[t]{3}{*}{ GR Ref } & Material & ${ }^{14} \mathrm{C}$ & S D & Lab No & \multirow[t]{3}{*}{ Reference } & \multirow[t]{3}{*}{ Comment } \\
\hline & & wood & 41150 & $\begin{array}{l}+1450 \\
-1250\end{array}$ & GrN 7999 & & \\
\hline & & wood & 53400 & $\begin{array}{r}+1700 \\
-1400\end{array}$ & GrN 8277 & & \\
\hline $\begin{array}{l}\text { City of Melbourne } \\
\text { Bay, King Island }\end{array}$ & e $77-535671$ & wood & 37500 & 1900 & NZ 349 & $\begin{array}{l}\text { Grant-Taylor \& } \\
\text { Rafter } 1962\end{array}$ & $\begin{array}{l}\text { Nothofagus in } \\
\text { alluvium below dune }\end{array}$ \\
\hline \multirow[t]{5}{*}{ Coal River } & $32-371763$ & charcoal & 1730 & 110 & Gak 1678 & $\begin{array}{l}\text { Kigoshiet al. } \\
1969\end{array}$ & alluvium \\
\hline & $32-364668$ & charcoal & 1990 & 100 & Gak 905 & & alluvial silty sand \\
\hline & $32-359693$ & $\begin{array}{l}\text { soil } \\
\text { carbonate }\end{array}$ & 2240 & 110 & Gak 2236 & & $\begin{array}{l}\text { pedal coating of } \\
\text { alluvium }\end{array}$ \\
\hline & $32-370662$ & charcoal & 5480 & 130 & Gak 2238 & & alluvial sand \\
\hline & $32-363668$ & charcoal & 33600 & $\begin{array}{r}+3400 \\
-2400\end{array}$ & Gak 906 & & $\begin{array}{l}\text { alluvial gravel } \\
\text { reworked }\end{array}$ \\
\hline \multirow[t]{4}{*}{ Curries River } & $25-960501$ & charcoal & 370 & 100 & SUA 1081 & $\begin{array}{l}\text { (Colhoun \& } \\
\text { van de Geer) }\end{array}$ & $\begin{array}{l}\text { Holocene \& } \\
\text { Pleistocene alluvial } \\
\text { deposits }\end{array}$ \\
\hline & & wood & 1460 & 80 & SUA 1082 & & \\
\hline & & wood & 1280 & 100 & SUA 1043 & & \\
\hline & & wood & 12570 & 240 & SUA 1083 & & \\
\hline \multirow[t]{2}{*}{ Denison River } & $02-048699$ & charcoal & 310 & 150 & ANU 2787 & $\begin{array}{l}\text { (Jones \& } \\
\text { Ranson) }\end{array}$ & $\begin{array}{l}\text { alluvial silt } 0.05 \mathrm{~m} \\
\text { below rainforest peat }\end{array}$ \\
\hline & & $\begin{array}{l}\text { plant } \\
\text { fragments }\end{array}$ & 13110 & 400 & SUA 1084 & & \\
\hline Narcissus River & I3-256484 & charcoal & 7650 & 250 & SUA 2079 & (Kiernan) & $\begin{array}{l}\text { basal } 0.2 \mathrm{~m} \text { of } \\
\text { alluvium on outwash } \\
\text { gravel }\end{array}$ \\
\hline $\begin{array}{l}\text { Native Hut } \\
\text { Rivulet }\end{array}$ & $32-352769$ & charcoal & 4160 & 160 & Gak 2237 & (Goede) & alluvium \\
\hline Oyster Creek & $32-118651$ & charcoal & 3840 & 95 & I 7931 & Wasson 1977 & $\begin{array}{l}\text { inset fill of alluvial } \\
\text { fan }\end{array}$ \\
\hline Parramore Creek & $32-148671$ & charcoal & 3575 & 95 & 17930 & Wasson 1977 & $\begin{array}{l}\text { inset fill of alluvial } \\
\text { fan }\end{array}$ \\
\hline \multirow[t]{2}{*}{ Pieman Dam } & $94-472742$ & wood & $>39000$ & & SUA 310 & Colhoun 1980 & $\begin{array}{l}\text { interglacial age } \\
\text { alluvium }\end{array}$ \\
\hline & & wood & $>54000$ & & GrN 7555 & & $\begin{array}{l}\text { same piece wood as } \\
\text { SUA } 310\end{array}$ \\
\hline \multirow[t]{5}{*}{ Rocky Cape } & $96-726741$ & $\begin{array}{l}\text { charred } \\
\text { wood }\end{array}$ & 26760 & 1360 & Gak 5153 & Colhoun $1977 \mathrm{~b}$ & $\begin{array}{l}\text { organic materials in } \\
\text { alluvial fan deposits }\end{array}$ \\
\hline & & wood & 28930 & $\begin{array}{r}+1970 \\
-1580\end{array}$ & Gak 5154 & & \\
\hline & & $\begin{array}{l}\text { charcoal \& } \\
\text { carb. wood }\end{array}$ & 33240 & $\begin{array}{r}+5610 \\
-3270\end{array}$ & Gak 5691 & & \\
\hline & & wood & 24090 & 1030 & Gak 5155 & & \\
\hline & & $\begin{array}{l}\text { charcoal \& } \\
\text { carb. wood }\end{array}$ & 32350 & $\begin{array}{r}+3680 \\
-2510\end{array}$ & Gak 5690 & & \\
\hline \multirow[t]{6}{*}{ Stanley River } & $94-565780$ & wood & 100 & 60 & SUA 5015 & $\begin{array}{l}\text { Francey et al. } \\
1984\end{array}$ & $\begin{array}{l}\text { logs of Lagaro- } \\
\text { strobos franklinii } \\
\text { from alluvium listed } \\
\text { chronologically }\end{array}$ \\
\hline & & wood & 120 & 60 & SUA 5016 & & \\
\hline & & wood & 630 & 60 & SUA 5021 & & \\
\hline & & wood & 1190 & 70 & SUA 5014 & & \\
\hline & & wood & 1210 & 70 & SUA 5013 & & \\
\hline & & wood & 1580 & 60 & SUA 5011 & & \\
\hline
\end{tabular}




\begin{tabular}{|c|c|c|c|c|c|c|c|}
\hline \multirow{3}{*}{ Site Name } & \multicolumn{6}{|c|}{ Fluvial Landforms and Deposits } & \multirow{3}{*}{ Comment } \\
\hline & GR Ref & Material & ${ }^{14} \mathrm{C}$ & $\mathbf{S ~ D}$ & Lab No & Reference & \\
\hline & & wood & 1980 & 60 & SUA 5012 & & \\
\hline & & wood & 2050 & 50 & SUA 5010 & & \\
\hline & & wood & 3680 & 40 & SUA 5023 & & \\
\hline & & wood & 3750 & 40 & SUA 5025 & & \\
\hline & & wood & 4090 & 40 & SUA 5027 & & \\
\hline & & wood & 4670 & 70 & SUA 5019 & & \\
\hline & & wood & 5500 & 50 & SUA 5020 & & \\
\hline & & wood & 6190 & 60 & SUA 5004 & & \\
\hline & & wood & 4080 & 70 & SUA 5018 & & $\begin{array}{l}\text { logs of Phyllocladus } \\
\text { asplenifolius }\end{array}$ \\
\hline & & wood & 4410 & 50 & SUA 5026 & & \\
\hline & & wood & 12390 & 80 & SUA 5022 & & $\begin{array}{l}\text { outer wood of } \\
\text { SUA } 5028\end{array}$ \\
\hline & & wood & 12870 & 90 & SUA 5028 & & $\begin{array}{l}\text { inner wood of } \\
\text { SUA } 5022\end{array}$ \\
\hline \multirow[t]{7}{*}{ Tea Tree Rivulet } & $42-639798$ & charcoal & 3040 & 90 & Gak 1677 & $\begin{array}{l}\text { Kigoshi et al. } \\
1969, \\
\text { Goede } 1973\end{array}$ & alluvium \\
\hline & & charcoal & 3850 & 90 & Gak 1148 & & alluvium \\
\hline & & wood & 4360 & 90 & Gak 1147 & & alluvium \\
\hline & & wood & 4435 & 110 & GX 99 & & altuvium \\
\hline & & charcoal & 5720 & 120 & Gak 1289 & & alluvium \\
\hline & & wood & 6200 & 200 & Gak 1146 & & $\begin{array}{l}\text { same horizon as } \\
\text { preceding date }\end{array}$ \\
\hline & & \multicolumn{5}{|c|}{ Glacial Landforms and Deposits } & \\
\hline Site Name & GR Ref & Material & ${ }^{14} \mathrm{C}$ & S D & Lab No & Reference & Comment \\
\hline $\begin{array}{l}\text { Conglomerate } \\
\text { Creek }\end{array}$ & 03-815406 & wood & 30050 & 2000 & ANU 2535 & (Colhoun) & $\begin{array}{l}\text { min. age of organic } \\
\text { bed between tills }\end{array}$ \\
\hline Crooked Lake & $12-410893$ & $\begin{array}{l}\text { detritus } \\
\text { mud }\end{array}$ & 5970 & 100 & SUA 1355 & $\begin{array}{l}\text { (Colhoun and } \\
\text { Macphail) }\end{array}$ & $\begin{array}{l}\text { min. age of mud in } \\
\text { channel outside } \\
\text { lateral moraine }\end{array}$ \\
\hline \multirow[t]{3}{*}{$\begin{array}{l}\text { Dante Site on } \\
\text { King River }\end{array}$} & $03-902459$ & wood & 18800 & 500 & ANU 2533 & $\begin{array}{l}\text { (Kiernan \& } \\
\text { Colhoun) }\end{array}$ & $\begin{array}{l}\text { twigs in base of } \\
\text { outwash silt and sand }\end{array}$ \\
\hline & & $\begin{array}{l}\text { Donatia } \\
\text { novae } \\
\text { zelandiae }\end{array}$ & 21180 & 370 & SUA 2154 & & $\begin{array}{l}\text { cushion in alpine } \\
\text { humus soil }\end{array}$ \\
\hline & & wood & 20100 & 470 & SUA 2155 & & $\begin{array}{l}\text { twigs in silt below } \\
\text { soil }\end{array}$ \\
\hline Fish River Road & $14-373746$ & charcoal & $>28000$ & & SUA 1938 & $\begin{array}{l}\text { (Hannan \& } \\
\text { Colhoun) }\end{array}$ & $\begin{array}{l}\text { charcoal in lateral } \\
\text { moraine }\end{array}$ \\
\hline Gormanston & $03-834410$ & wood & 1560 & 250 & ANU 2534 & $\begin{array}{l}\text { (Kiernan \& } \\
\text { Colhoun) }\end{array}$ & $\begin{array}{l}\text { wood intrusive in } \\
\text { varved clays }\end{array}$ \\
\hline Lake Nancy & $03-032207$ & $\begin{array}{l}\text { wood \& leaf } \\
\text { fragments }\end{array}$ & 8720 & 220 & Gak 671 & Peterson 1968 & min. age deglaciation \\
\hline \multirow[t]{4}{*}{ Linda Creek } & $03-835422$ & wood & 26480 & 800 & W 323 & Gill 1956 & $\begin{array}{l}\text { wood in varved } \\
\text { clays }\end{array}$ \\
\hline & & wood & $>40000$ & NZ 348 & $\begin{array}{l}\text { Grant-Taylor } \\
\text { \& Rafter } \\
1962,1963\end{array}$ & $\begin{array}{l}\text { clayey sands of } \\
\text { preglacial age }\end{array}$ & \\
\hline & & wood & 27800 & 700 & ANU $2480 \mathrm{~A}$ & Colhoun 1985 & wood in glacial sands \\
\hline & & $\alpha$ cellulose & 23100 & 600 & ANU $2480 B$ & & $\begin{array}{l}\text { same wood as } \\
\text { ANU } 2480 \mathrm{~A}\end{array}$ \\
\hline Mackintosh Dam & $04-872825$ & charcoal & $>35000$ & & SUA 1289 & Colhoun 1985 & till $>{ }^{4} \mathrm{C}$ limit \\
\hline
\end{tabular}


Lake, Swamp and Peat Deposits

\begin{tabular}{|c|c|c|c|c|c|c|c|}
\hline Site Name & GR Ref & Material & ${ }^{14} \mathrm{C}$ & S D & Lab No & Reference & Comment \\
\hline Adamsons Peak & $21-867004$ & $\begin{array}{l}\text { carbon } \\
\text { fragments }\end{array}$ & 9080 & 200 & I 7571 & $\begin{array}{l}\text { Macphail \& } \\
\text { Peterson } 1975\end{array}$ & $2.5 \mathrm{~m}$ in core in cirque \\
\hline \multirow[t]{4}{*}{ Badger Beach } & $25-740507$ & wood & 7380 & 100 & NZ 1219 & $\begin{array}{l}\text { Fish \& Yaxley } \\
1966\end{array}$ & $\begin{array}{l}\text { tree stump in } \\
\text { intertidal peat bed }\end{array}$ \\
\hline & & peat & 8020 & 140 & Gak 5622 & (Chick) & peat bed at high tide \\
\hline & & peat & 7850 & 140 & Gak 5621 & & peat bed at mid tide \\
\hline & & peat & 31960 & $\begin{array}{r}+3400 \\
-2380\end{array}$ & Gak 5620 & & $\begin{array}{l}\text { wave-worn peat } \\
\text { masses derived from } \\
\text { below low tide }\end{array}$ \\
\hline \multirow[t]{3}{*}{ Beatties Tarn } & $22-705752$ & $\begin{array}{l}\text { Eucalyptus } \\
\text { leaves }\end{array}$ & 9050 & 200 & Gak 878 & Macphail 1979 & $\begin{array}{l}1.4 \mathrm{~m} \text { in core in cirque } \\
\text { lake }\end{array}$ \\
\hline & & lake mud & 7850 & 150 & SUA 324 & & $1.4-1.65 \mathrm{~m}$ \\
\hline & & lake mud & 11420 & 205 & SUA 325 & & $3.2-3.4 \mathrm{~m}$ \\
\hline $\begin{array}{l}\text { Ben Lomond, } \\
\text { Rodway Valley }\end{array}$ & $44-557991$ & $\begin{array}{l}\text { wood } \\
\text { fragments }\end{array}$ & 3080 & 90 & Gak 670 & Caine 1983 & $\begin{array}{l}\text { peat over } \\
\text { blockstream; } \\
\text { formerly Talus Valley }\end{array}$ \\
\hline Bird River Track & $03-837110$ & wood & 1290 & 70 & SUA 5017 & $\begin{array}{l}\text { Francey et al. } \\
1984\end{array}$ & $\begin{array}{l}\text { log of Lagarostrobos } \\
\text { franklinii }\end{array}$ \\
\hline Borradaile Plains & $14-318857$ & peat & 10480 & 180 & Gak 785 & (Peterson) & $\begin{array}{l}\text { peat from depression } \\
\text { in glacial drift }\end{array}$ \\
\hline \multirow[t]{2}{*}{ Broad Valley } & $22-661791$ & sedges & 3050 & 104 & NSW 29B & Caine 1968 & Im depth \\
\hline & $22-659797$ & sedges & 3500 & 80 & NSW 37B & & $1.25 \mathrm{~m} \mathrm{depth}$ \\
\hline \multirow[t]{3}{*}{$\begin{array}{l}\text { Broadmeadows } \\
\text { Swamp }\end{array}$} & $36-385733$ & peat & 11410 & 770 & Gak 5969 & $\begin{array}{l}\text { (van de Geer } \\
\text { et al.) }\end{array}$ & $\begin{array}{l}\text { Holocene \& late } \\
\text { Pleistocene swamp } \\
\text { deposits at } 0.70 \\
0.85 \& 1.0 \mathrm{~m}\end{array}$ \\
\hline & & peat & 15100 & 750 & Gak 7556 & & \\
\hline & & peat & 27600 & $\begin{array}{r}+1700 \\
-1430\end{array}$ & Gak 6324 & & \\
\hline \multirow[t]{3}{*}{ Brown Marsh } & 23-643305 & peat & 3490 & 90 & ANU 2744 & $\begin{array}{l}\text { Macphail \& } \\
\text { Hope } 1985\end{array}$ & $\begin{array}{l}2.7 \mathrm{~m} \text { depth in } \\
\text { core } 2\end{array}$ \\
\hline & & peat & 8575 & 125 & I 9558 & Macphail 1979 & $3.1 \mathrm{~m}$ \\
\hline & & peat & 4930 & 180 & Gak 984 & (Peterson) & $\begin{array}{l}5.1 \mathrm{~m} \text { depth in } \\
\text { core } 1\end{array}$ \\
\hline \multirow[t]{3}{*}{$\begin{array}{l}\text { Camerons } \\
\text { Lagoon }\end{array}$} & 24-732545 & $\begin{array}{l}\text { algal } \\
\text { gyttja }\end{array}$ & 2670 & 70 & ANU 3974 & (Thomas) & $0.2-0.28 \mathrm{~m}$ in core \\
\hline & & & 7500 & 130 & ANU 3976 & & $0.32-0.37 \mathrm{~m}$ \\
\hline & & & 7750 & 150 & ANU 2739 & & $0.52-0.59 \mathrm{~m}$ \\
\hline Darwin Crater & $03-892153$ & peat & $>51000$ & & GrN 7694 & $\begin{array}{l}\text { (Colhoun \& } \\
\text { van de Geer) }\end{array}$ & $\begin{array}{l}6.32-6.38 \mathrm{~m} \text { in } 1972 \\
\text { core }\end{array}$ \\
\hline \multirow[t]{4}{*}{ Dublin Bog } & $14-371815$ & org. mud & 8920 & 140 & SUA 2191 & $\begin{array}{l}\text { (Colhoun \& } \\
\text { Hannan) }\end{array}$ & $4.16-4.21 \mathrm{~m}$ in core \\
\hline & & org. mud & 11710 & 190 & SUA 2190 & & $6.24-6.29 \mathrm{~m}$ \\
\hline & & org. mud & 13150 & 240 & SUA 2189 & & $7.34-7.46 \mathrm{~m}$ \\
\hline & & $\begin{array}{l}\text { charcoal in } \\
\text { lake clay }\end{array}$ & 13400 & 600 & SUA 2188 & & $8.30-8.46 \mathrm{~m}$ \\
\hline Dyes Marsh & $13-485385$ & peat & 4930 & 180 & Gak 784 & (Derbyshire) & $5.2 \mathrm{~m}$ depth \\
\hline \multirow[t]{6}{*}{ Eagle Tarn } & $22-664745$ & org. mud & 9960 & 300 & SUA 323 & Macphail 1979 & $3.9-4.1 \mathrm{~m}$ \\
\hline & & org. mud & 11400 & 240 & I 7685 & & $4.4-4.6 \mathrm{~m}$ \\
\hline & & org. mud & 6300 & 400 & ANU 3106 & (Green) & $2.1-2.2 \mathrm{~m}$ in core \\
\hline & & org. mud & 6420 & 380 & ANU 3108 & & $2.5-2.6 \mathrm{~m}$ \\
\hline & & org. mud & 7440 & 500 & ANU 3112 & & $3.3-3.4 \mathrm{~m}$ \\
\hline & & org. mud & 7460 & 350 & ANU 3113 & & $3.5-3.6 \mathrm{~m}$ \\
\hline
\end{tabular}




\begin{tabular}{|c|c|c|c|c|c|c|c|}
\hline \multirow{6}{*}{ Site Name } & \multirow{6}{*}{ GR Ref } & \multicolumn{5}{|c|}{ Lake, Swamp and Peat Deposits } & \multirow[b]{2}{*}{$\begin{array}{l}\text { Comment } \\
3.7-3.8 \mathrm{~m}\end{array}$} \\
\hline & & $\begin{array}{l}\text { Material } \\
\text { org. mud }\end{array}$ & $\begin{array}{r}{ }_{9}^{14} \mathrm{C} \\
9650\end{array}$ & $\begin{array}{r}\text { S D } \\
850\end{array}$ & $\begin{array}{l}\text { Lab No } \\
\text { ANU } 3114\end{array}$ & Reference & \\
\hline & & org. mud & 8.300 & 490 & ANU 3115 & & $3.9-4.0 \mathrm{~m}$ \\
\hline & & org. mud & 6340 & 190 & ANU 3116 & & $4.1-4.2 \mathrm{~m}$ \\
\hline & & org. mud & 7460 & 380 & ANU 3117 & & $4.3-4.4 \mathrm{~m}$ \\
\hline & & org. mud & 12960 & 950 & ANU 3118 & & $4.5-4.6 \mathrm{~m}$ \\
\hline Gordon Road & $12-510657$ & wood & 33240 & $\begin{array}{r}+3370 \\
-2370\end{array}$ & Gak 5625 & (Colhoun) & $\begin{array}{l}\text { base of ice-pushed } \\
\text { organic clays. } \\
\text { Probably a minimum } \\
\text { age. }\end{array}$ \\
\hline Hartz Lake & $21-805128$ & peat & 2930 & 100 & SUA 1357 & $\begin{array}{l}\text { (Colhoun \& } \\
\text { Macphail) }\end{array}$ & $0.5-0.59 \mathrm{~m}$ \\
\hline \multirow{7}{*}{ Henty Bridge } & \multirow{7}{*}{$94-734495$} & lake clay & 4100 & 200 & SUA 1944 & & $\begin{array}{l}0.71-0.9 \mathrm{~m} \text { in lake } \\
\text { clay of core }\end{array}$ \\
\hline & & lake clay & Modern & & SUA 1945 & & $1.6-1.89 \mathrm{~m}$ \\
\hline & & org. clay & 23640 & 1030 & Gak 5597 & Colhoun 1979 & $\begin{array}{l}\text { lake clays and slope } \\
\text { deposits on till }\end{array}$ \\
\hline & & org. clay & 25660 & 1200 & Gak 5596 & & \\
\hline & & wood & $>34600$ & & Gak 5595 & & \\
\hline & & wood & $>34190$ & & Gak 6294 & & \\
\hline & & wood & 23860 & 890 & Gak 5594 & & \\
\hline $\begin{array}{l}\text { Henty Surface } \\
\text { Swamp }\end{array}$ & $04-786537$ & org. mud & $>4380$ & & Gak 6292 & (Colhoun) & $1.98-2.0 \mathrm{~m}$ in core \\
\hline Hogsback & $21-874945$ & wood & 750 & 60 & SUA 2110 & (Podger) & $\begin{array}{l}\text { Telopea rootstock at } \\
0.5 \mathrm{~m} \text { depth in peat }\end{array}$ \\
\hline Hogsback Plain & $21-863960$ & charcoal & 11600 & 100 & SUA 2109 & (Podger) & base peat at $1.2 \mathrm{~m}$ \\
\hline $\begin{array}{l}\text { Homestead } \\
\text { Swamp } \\
\text { Hunter Island }\end{array}$ & $86-094110$ & peat & 4100 & 80 & ANU 2532 & (Hope) & sandsheet stabilised \\
\hline $\begin{array}{l}\text { James Tarn, } \\
\text { Mount Field }\end{array}$ & $22-641757$ & org. mud & 8280 & 460 & Gak 1158 & $\begin{array}{l}\text { Macphail \& } \\
\text { Peterson } 1975\end{array}$ & $1.5 \mathrm{~m}$ in core \\
\hline \multirow{3}{*}{$\begin{array}{l}\text { Killiecrankie Bay, } \\
\text { Flinders Island }\end{array}$} & $48-719900$ & sandy peat & 12550 & 390 & Gak 8261 & (Ladd) & $2.45 \mathrm{~m}$ in core \\
\hline & & peat & $>33890$ & & Gak 8923 & & $3.2 \mathrm{~m}$ \\
\hline & & peat & 29630 & 3030 & Gak 8922 & & $4.25-4.28 \mathrm{~m}$ \\
\hline $\begin{array}{l}\text { Killiecrankie } \\
\text { Swamp, } \\
\text { Flinders Island }\end{array}$ & $48-715898$ & wood & 550 & 140 & Gak 8263 & Ladd 1981 & $2.0 \mathrm{~m}$ depth \\
\hline Lagoon of Islands & $23-940390$ & peat & 7430 & 150 & unknown & (Jackson) & base peat \\
\hline Lake Tiberias & $33-300020$ & peat & 9550 & 200 & Gak 2239 & $\begin{array}{l}\text { Macphail \& } \\
\text { Jackson } 1978\end{array}$ & $\begin{array}{l}\text { base of organic } \\
\text { deposit } \sim 2 \mathrm{~m} \text { depth }\end{array}$ \\
\hline \multirow{2}{*}{ Lake Vera } & \multirow[t]{2}{*}{$03-078193$} & org. clay & 6950 & 175 & I 9557 & Macphail 1979 & $2.08-2.13 \mathrm{~m}$ in core \\
\hline & & org. clay & 11530 & 240 & I 7683 & & $2.7-2.8 \mathrm{~m}$ \\
\hline $\begin{array}{l}\text { Lake Wurawina, } \\
\text { Upper Cirque }\end{array}$ & $12-405900$ & peat & 10360 & 170 & SUA 1354 & $\begin{array}{l}\text { (Colhoun \& } \\
\text { Macphail) }\end{array}$ & $1.65-1.75 \mathrm{~m}$ in core \\
\hline $\begin{array}{l}\text { Laughing Jack } \\
\text { Lagoon }\end{array}$ & $13-415320$ & wood & 810 & 60 & SUA 1957 & (Kiernan) & $0.45-0.5 \mathrm{~m}$ \\
\hline \multirow{3}{*}{$\begin{array}{l}\text { Lower Mackin- } \\
\text { tosh Valley }\end{array}$} & \multirow{3}{*}{$04-869822$} & wood & 1540 & 60 & SUA 1958 & & $\sim 1 \mathrm{~m}$ depth in silt \\
\hline & & wood & 36200 & $\begin{array}{r}+3400 \\
-2400\end{array}$ & SUA 1287 & $\begin{array}{l}\text { (Colhoun \& } \\
\text { Sansom) }\end{array}$ & $0.9 \mathrm{~m}$ in section \\
\hline & & charcoal & $>31700$ & & SUA 1288 & & $1.7-1.75 \mathrm{~m}$ in section \\
\hline $\begin{array}{l}\text { Mickelthwaite } \\
\text { Marsh }\end{array}$ & $05-846106$ & peat & 7030 & 110 & I 11863 & $\begin{array}{l}\text { (Colhoun \& } \\
\text { van de Geer) }\end{array}$ & $0.85-0.9 \mathrm{~m}$ in core \\
\hline Middle Patriarch & 58-009719 & peat & 940 & 110 & Gak 8921 & (Ladd) & $0.65-0.7 \mathrm{~m}$ in core \\
\hline
\end{tabular}


Lake, Swamp and Peat Deposits

\begin{tabular}{|c|c|c|c|c|c|c|c|}
\hline \\
\hline \multirow[t]{3}{*}{ Site Name } & \multirow[t]{3}{*}{ GR Ref } & Material & ${ }^{14} \mathrm{C}$ & S D & Lab No & \multirow[t]{3}{*}{ Reference } & Comment \\
\hline & & peat & 5920 & 150 & Gak 8920 & & $1.4-1.45 \mathrm{~m}$ \\
\hline & & peat & 10110 & 420 & Gak 8258 & & $1.7-1.75 \mathrm{~m}$ \\
\hline $\begin{array}{l}\text { Mount Gould } \\
\text { Plateau }\end{array}$ & $14-220512$ & org. silt & 7920 & 250 & SUA 2080 & (Kiernan) & $1.6 \mathrm{~m}$ in core \\
\hline Mount McCall & $03-956100$ & wood & 2740 & 60 & SUA 2108 & (Podger) & $\begin{array}{l}\text { Athrotaxis wood } \\
\text { partly buried }\end{array}$ \\
\hline $\begin{array}{l}\text { Mount Strzelecki, } \\
\text { near summit }\end{array}$ & $57-914496$ & peat & 1510 & 130 & Gak 8260 & (Ladd) & $0.84-0.86 \mathrm{~m}$ on rock \\
\hline \multirow[t]{10}{*}{ Mowbray Swamp } & \multirow[t]{10}{*}{$96-365760$} & marl & $>37760$ & & Y $148 / 1$ & $\begin{array}{l}\text { Barendsen et al. } \\
1957\end{array}$ & $0.6 \mathrm{~m}$ depth in swamp \\
\hline & & peat & $>37760$ & & Y $148 / 2$ & & $0.6-1.2 \mathrm{~m}$ \\
\hline & & tree stump & 47500 & $\begin{array}{l}+2700 \\
-2000\end{array}$ & GrN 7481 & \multirow[t]{8}{*}{$\begin{array}{l}\text { (van de Geer } \\
\text { et al.) }\end{array}$} & $\begin{array}{l}1.35 \mathrm{~m} \text { in spring } \\
\text { mound }\end{array}$ \\
\hline & & root stump & 36300 & 700 & GrN 8606 & & $0.85 \mathrm{~m}$ depth in pit \\
\hline & & $\begin{array}{l}\text { humic } \\
\text { extract }\end{array}$ & 34100 & 700 & GrN 8646 & & of $\mathrm{GrN} 8606$ \\
\hline & & peat & 46400 & $\begin{array}{r}+1300 \\
-1100\end{array}$ & GrN 9341 & & $1.075-1.125 \mathrm{~m}$ \\
\hline & & $\begin{array}{l}\text { humic } \\
\text { extract }\end{array}$ & 45200 & $\begin{array}{r}+2600 \\
-2000\end{array}$ & GrN 9765 & & of $\mathrm{GrN} 9341$ \\
\hline & & root stump & $>52000$ & & GrN 9342 & & $1.2-1.3 \mathrm{~m}$ \\
\hline & & root stump & (52 220 & $350)$ & GrN 9742 & & $\begin{array}{l}\text { Same sample as } \\
\text { GrN } 9342\end{array}$ \\
\hline & & $\begin{array}{l}\text { humic } \\
\text { extract }\end{array}$ & (51 300 & $\begin{array}{l}+4400) \\
-2800)\end{array}$ & GrN 9767 & & of $\mathrm{GrN} 9742$ \\
\hline \multirow[t]{7}{*}{ Ooze Lake } & \multirow[t]{7}{*}{$20-758837$} & lake clay & 5440 & 90 & SUA 1939 & \multirow[t]{3}{*}{$\begin{array}{l}\text { (Colhoun \& } \\
\text { Macphail) }\end{array}$} & $1.0-1.2 \mathrm{~m}$ in core \\
\hline & & lake clay & 8870 & 130 & SUA 1940 & & $2.0-2.2 \mathrm{~m}$ \\
\hline & & lake clay & 10390 & 160 & SUA 1941 & & $3.0-.3 .2 \mathrm{~m}$ \\
\hline & & lake clay & 10670 & 170 & SUA 1942 & \multirow[t]{4}{*}{$\begin{array}{l}\text { Macphail \& } \\
\text { Colhoun } 1985\end{array}$} & $4.0-4.2 \mathrm{~m}$ \\
\hline & & lake clay & 12100 & 160 & SUA 1356 & & $4.77-5.07 \mathrm{~m}$ \\
\hline & & lake clay & 12590 & 230 & SUA 1943 & & $5.5-5.7 \mathrm{~m}$ \\
\hline & & lake clay & 17700 & 400 & SUA 1359 & & $6.4-6.65 \mathrm{~m}$ \\
\hline \multirow[t]{3}{*}{ Phillips Creek } & \multirow[t]{3}{*}{$45-512058$} & wood & 8475 & 85 & Beta 1885 & \multirow[t]{2}{*}{ Caine 1983} & $0.8 \mathrm{~m}$ depth in core 2 \\
\hline & & lake clay & 9535 & 165 & Beta 1886 & & $2.25 \mathrm{~m}$ \\
\hline & & $\begin{array}{l}\text { clay with } \\
\text { charcoal }\end{array}$ & 6270 & 760 & ANU 2512 & (Noble) & $2.27 \mathrm{~m}$ core 1 \\
\hline \multirow{4}{*}{$\begin{array}{l}\text { Poets Hill Lake, } \\
\text { formerly referred } \\
\text { to as Inter- } \\
\text { moraine Lake }\end{array}$} & \multirow[t]{4}{*}{$04-807508$} & org. mud & 3600 & 180 & SUA 1394 & \multirow[t]{4}{*}{ Colhoun 1979} & $0.505-0.54 \mathrm{~m}$ core 2 \\
\hline & & org. mud & 10150 & 220 & SUA 1395 & & $1.82-1.86 \mathrm{~m}$ core 2 \\
\hline & & org. mud & 7410 & 400 & SUA 1396 & & $\begin{array}{l}2.85-2.9 \mathrm{~m} \text { core } 2 \\
\text { believed unreliable }\end{array}$ \\
\hline & & org. mud & 11420 & 770 & Gak 6297 & & $2.9-2.95 \mathrm{~m}$ core 1 \\
\hline \multirow[t]{5}{*}{ Pulbeena Swamp } & \multirow[t]{5}{*}{$96-436724$} & peat & 13690 & 550 & Y $229 / 1$ & \multirow[t]{2}{*}{$\begin{array}{l}\text { Barendsen et al. } \\
1957\end{array}$} & $\begin{array}{l}0.62-0.8 \mathrm{~m} \text { depth in } \\
\text { swamp }\end{array}$ \\
\hline & & shell marl & 27900 & 2000 & Y $229 / 2$ & & $1.70 \mathrm{~m}$ \\
\hline & & wood & 54200 & $\begin{array}{r}+11000 \\
-4500\end{array}$ & GrN 7322 & $\begin{array}{l}\text { Banks et al. } \\
1976\end{array}$ & $\begin{array}{l}2.3 \mathrm{~m} \text { in profile of } \\
1975\end{array}$ \\
\hline & & $\begin{array}{l}\text { humified } \\
\text { peat }\end{array}$ & 11370 & 70 & GrN 7881 & \multirow[t]{2}{*}{$\begin{array}{l}\text { Colhoun et al. } \\
1982\end{array}$} & $\begin{array}{l}0.6 \mathrm{~m} \text { depth in section } \\
\text { of } 1976\end{array}$ \\
\hline & & $\begin{array}{l}\text { humified } \\
\text { peat }\end{array}$ & 14980 & 80 & GrN 7688 & & $0.75 \mathrm{~m}$ \\
\hline
\end{tabular}




\begin{tabular}{|c|c|c|c|c|c|c|c|}
\hline \multirow{3}{*}{ Site Name } & \multicolumn{6}{|c|}{ Lake, Swamp and Peat Deposits } & \multirow[b]{2}{*}{ Comment } \\
\hline & GR Ref & Material & ${ }^{14} \mathrm{C}$ & $\mathbf{S} \mathbf{D}$ & Lab No & Reference & \\
\hline & & peat & 16590 & 110 & GrN 7882 & & $0.85 \mathrm{~m}$ \\
\hline & & $\begin{array}{l}\text { humified } \\
\text { peat }\end{array}$ & 22130 & 180 & GrN 7689 & & $1.15 \mathrm{~m}$ \\
\hline & & $\begin{array}{l}\text { charred } \\
\text { root stump }\end{array}$ & 44700 & 1500 & GrN 7690 & & $1.65 \mathrm{~m}$ \\
\hline & & $\begin{array}{l}\text { charred } \\
\text { root stump }\end{array}$ & 42200 & 800 & GrN 9458 & & $1.65 \mathrm{~m}$ \\
\hline & & root stump & 48400 & $\begin{array}{l}+1900 \\
-1600\end{array}$ & GrN 7691 & & $2.0 \mathrm{~m}$ \\
\hline & & root stump & 53400 & $\begin{array}{r}+3700 \\
-2500\end{array}$ & GrN 9459 & & $2.0 \mathrm{~m}$ \\
\hline & & root stump & $(42700$ & $900)$ & GrN 8589 & & $2.05-2.15 \mathrm{~m}$ \\
\hline & & $\begin{array}{l}\text { humic } \\
\text { extract }\end{array}$ & $(41100$ & $800)$ & GrN 8636 & & of $\mathrm{GrN} 8589$ \\
\hline & & root stump & 41450 & 700 & GrN 9438 & & \\
\hline & & & $(42620$ & 200) & GrN 9483 & & $\begin{array}{l}\text { same sample as } \\
\text { GrN } 9438\end{array}$ \\
\hline & & root stump & $(48200$ & $250)$ & GrN 8526 & & $2.55-2.65 \mathrm{~m}$ \\
\hline & & $\begin{array}{l}\text { humic } \\
\text { extract }\end{array}$ & $(47500$ & $800)$ & GrN 8626 & & of GrN 8526 \\
\hline & & root stump & $(49250$ & $300)$ & GrN 8754 & & $3.2-3.3 \mathrm{~m}$ \\
\hline & & $\begin{array}{l}\text { humic } \\
\text { extract }\end{array}$ & $(47600$ & $\begin{array}{l}+1900) \\
(-1500)\end{array}$ & GrN 8627 & & of GrN 8754 \\
\hline & & $\begin{array}{l}\text { peat in } \\
\text { shell-marl }\end{array}$ & $>55000$ & & GrN 9798 & & $4.2-4.25 \mathrm{~m}$ \\
\hline & & $\begin{array}{l}\text { peat in } \\
\text { shell-marl }\end{array}$ & $(55200$ & $500)$ & GrN 9905 & & $\begin{array}{l}\text { same sample as } \\
\text { GrN } 9798\end{array}$ \\
\hline & & shell-marl & 42500 & 1100 & GrN 9844 & & $\begin{array}{l}\text { same horizon as } \\
\text { GrN } 9789\end{array}$ \\
\hline \multirow[t]{2}{*}{ Sundown Point } & $85-044445$ & peat & 420 & 60 & ANU 2531 & $\begin{array}{l}\text { (Hope \& } \\
\text { Ranson) }\end{array}$ & $0.21-0.27 \mathrm{~m}$ \\
\hline & & $\begin{array}{l}\text { peat \& org. } \\
\text { mud }\end{array}$ & 3300 & 80 & ANU 2530 & & $\begin{array}{l}0.8-0.9 \mathrm{~m}, \\
\text { stabilisation coastal } \\
\text { dune }\end{array}$ \\
\hline Scotts Peak Dam & $11-445235$ & carb. wood & $>33600$ & & Gak 5624 & (Colhoun) & $\begin{array}{l}\text { wood in ice-pushed? } \\
\text { lake clays }\end{array}$ \\
\hline \multirow[t]{3}{*}{ Tarraleah } & $13-557162$ & peat & 7000 & 160 & ANU 2496 & Macphail 1984 & $\begin{array}{l}0.4-0.5 \mathrm{~m} \text { depth in } \\
\text { core }\end{array}$ \\
\hline & & peat & 7970 & 180 & ANU 2745 & & $1.1-1.2 \mathrm{~m}$ \\
\hline & & org. clay & 9080 & 195 & I 9559 & & $2.5-2.54 \mathrm{~m}$ \\
\hline \multirow[t]{6}{*}{$\begin{array}{l}\text { Tullabardine } \\
\text { Creek }\end{array}$} & $96-879843$ & peat & 8030 & 110 & SUA 2185 & $\begin{array}{l}\text { (Colhoun } \& \\
\text { van de Geer) }\end{array}$ & $\begin{array}{l}1.2 \mathrm{~m} \text { depth in } \\
\text { monolith }\end{array}$ \\
\hline & & peat & 11060 & 210 & SUA 2186 & & $1.35 \mathrm{~m}$ \\
\hline & & wood & 11660 & 150 & SUA 1044 & & $1.6 \mathrm{~m}$ in section \\
\hline & & org. soil & 21250 & 270 & SUA 1045 & & $1.9-2.1 \mathrm{~m}$ \\
\hline & & wood & 31500 & 900 & SUA 1046 & & $\begin{array}{l}3.05-3.1 \mathrm{~m} \text { in } \\
\text { monolith }\end{array}$ \\
\hline & & wood & $>43800$ & & SUA 1047 & & $3.8 \mathrm{~m}$ \\
\hline Tyndall Plateau & $96-824545$ & org. clay & 9050 & 120 & SUA 1358 & $\begin{array}{l}\text { (Colhoun \& } \\
\text { Macphail) }\end{array}$ & $0.82-0.94 \mathrm{~m}$ in core \\
\hline
\end{tabular}




\begin{tabular}{|c|c|c|c|c|c|c|c|}
\hline \multicolumn{8}{|c|}{ Lake, Swamp and Peat Deposits } \\
\hline Site Name & GR Ref & Material & ${ }^{14} \mathrm{C}$ & S D & Lab No & Reference & Comment \\
\hline \multirow[t]{3}{*}{$\begin{array}{l}\text { Unnamed Tarn, } \\
\text { Mount Field }\end{array}$} & $22-640758$ & org. mud & 3570 & 115 & 1750 & (Peterson) & $0.35-0.4 \mathrm{~m}$ \\
\hline & & org. mud & 9590 & 240 & I 8007 & $\begin{array}{l}\text { Macphail \& } \\
\text { Peterson } 1975\end{array}$ & $1.5 \mathrm{~m}$ in core \\
\hline & & org. mud & 9725 & 180 & 18008 & & $3.0 \mathrm{~m}$ \\
\hline \multicolumn{8}{|c|}{ Slope Deposits } \\
\hline Site Name & GR Ref & Material & ${ }^{14} \mathrm{C}$ & S D & Lab No & Reference & Comment \\
\hline Barilla Hills & $32-343564$ & charcoal & 6490 & 100 & UB 919 & (Stephens) & $\begin{array}{l}\text { silt with charcoal } \\
\text { below mudstone } \\
\text { slope deposits }\end{array}$ \\
\hline $\begin{array}{l}\text { Ben Lomond, } \\
\text { Pig Run Creek }\end{array}$ & $44-500037$ & charcoal & 3940 & 110 & Beat 2109 & Caine 1983 & \\
\hline Chester Rivulet & $04-784795$ & charcoal & 18190 & 340 & SUA 1042 & (Colhoun) & $\begin{array}{l}\text { in soil at } 5.7 \mathrm{~m} \text { depth } \\
\text { in scree }\end{array}$ \\
\hline Gellibrand Point & $32-332428$ & carb. wood & $>37000$ & & SUA 309 & Colhoun 1975 & $\begin{array}{l}\text { strongly weathered } \\
\text { chemically }\end{array}$ \\
\hline Hardstaff Creek & $15-195367$ & charcoal & 13870 & 820 & Gak 5968 & (Colhoun) & $1.9 \mathrm{~m}$ depth in scree \\
\hline Mathinna Plains & $45-712145$ & charcoal & 2340 & 90 & Gak 669 & Caine 1978 & at $2 \mathrm{~m}$ depth in scree \\
\hline \multirow[t]{6}{*}{ Remarkable Cave } & $41-686178$ & wood & 40000 & & SUA 347 & Colhoun $1977 \mathrm{c}$ & $\begin{array}{l}\text { in } 17 \mathrm{~m} \text { of slope } \\
\text { deposits }\end{array}$ \\
\hline & & $\begin{array}{l}\text { wood \& } \\
\text { plant } \\
\text { detritus }\end{array}$ & $>40000$ & & SUA 348 & & \\
\hline & & wood & $>40000$ & & SUA 349 & & \\
\hline & & peaty clay & $>37000$ & & SUA 389 & & \\
\hline & & $\begin{array}{l}\text { charcoal \& } \\
\text { wood }\end{array}$ & $>37000$ & & SUA 391 & & \\
\hline & & charcoal & 29050 & 830 & SUA 154 & & $\begin{array}{l}\text { contaminated by } \\
\text { humic acid }\end{array}$ \\
\hline Rokeby & $32-389493$ & wood & 470 & 90 & Gak? & $\begin{array}{l}\text { (Stephens \& } \\
\text { van de Geer) }\end{array}$ & slope deposits on cliff \\
\hline Sayers Hill & $15-365378$ & charcoal & $>30600$ & & Gak 5590 & Colhoun 1976 & $\begin{array}{l}\text { at } 5 \mathrm{~m} \text { depth in slope } \\
\text { deposit }\end{array}$ \\
\hline \multicolumn{8}{|c|}{ Soils and Palaeosols } \\
\hline Site Name & GR Ref & Material & ${ }^{14} \mathrm{C}$ & S D & Lab No & Reference & Comment \\
\hline Badger Beach & $25-694471$ & org. clay & 15900 & 510 & Gak 5619 & (Chick) & $\begin{array}{l}\text { Podzol below dune } \\
\text { and on beach sands }\end{array}$ \\
\hline $\begin{array}{l}\text { Ben Lomond, } \\
\text { Land of Little } \\
\text { Sticks }\end{array}$ & $44-537029$ & $\begin{array}{l}\text { peaty soil } \\
\text { with } \\
\text { charcoal }\end{array}$ & 4940 & 90 & ANU 2513 & Caine 1983 & $0.76-0.77 \mathrm{~m}$ depth \\
\hline Ossians Throne & $44-580992$ & org. soil & 1250 & 70 & Beta 2110 & Caine 1983 & buried A horizon \\
\hline $\begin{array}{l}\text { Ben Lomond, } \\
\text { Road }\end{array}$ & $44-518050$ & org. soil & 19410 & 330 & Beta 1887 & Caine 1983 & $\begin{array}{l}\text { covered by blocky } \\
\text { mantle }\end{array}$ \\
\hline Fern Tree & $32-204471$ & charcoal & $>40000$ & & I 8155 & Colhoun 1975 & buried Bt horizon \\
\hline Florentine Valley & $12-553815$ & charcoal & 14200 & 700 & Gak 486 & Davies 1974 & $\begin{array}{l}\text { Podzol A horizon } \\
\text { below solifluction }\end{array}$ \\
\hline \multirow[t]{2}{*}{ Linda Creek } & $03-835422$ & wood & $>40000$ & & R 488 & (Banks) & $\begin{array}{l}\text { Phyllocladus stump } \\
\text { below glacial sands }\end{array}$ \\
\hline & & wood & $>48500$ & & ANU 3413 & $\begin{array}{l}\text { (Colhoun \& } \\
\text { Kiernan) }\end{array}$ & $\begin{array}{l}\text { Phyllocladus stump } \\
\text { below glacial sands }\end{array}$ \\
\hline
\end{tabular}




\begin{tabular}{|c|c|c|c|c|c|c|c|c|}
\hline \multicolumn{9}{|c|}{ Soils and Palaeosols } \\
\hline Site Name & \multicolumn{2}{|c|}{ GR Ref } & Material & ${ }^{14} \mathrm{C}$ & S D & Lab No & Reference & Comment \\
\hline \multirow[t]{2}{*}{$\begin{array}{l}\text { Monpeelyata } \\
\text { Canal }\end{array}$} & \multirow{2}{*}{\multicolumn{2}{|c|}{$23-730385$}} & $\begin{array}{l}\text { plant } \\
\text { detritus }\end{array}$ & 2900 & 80 & Gak 1020 & (Derbyshire) & $\begin{array}{l}\text { Astelia alpina below } \\
\text { solfluction but on } \\
\text { same level as Gak } \\
1163 \text {, date suspect }\end{array}$ \\
\hline & & & charcoal & 30400 & 2300 & Gak 1163 & Derbyshire 1968 & $\begin{array}{l}\text { soil below solifluction } \\
\text { deposit }\end{array}$ \\
\hline Mount Albert & \multicolumn{2}{|c|}{$45-742200$} & $\begin{array}{l}\text { wood \& } \\
\text { charcoal }\end{array}$ & 3520 & 100 & Gak 668 & Caine 1978 & $\begin{array}{l}\text { buried Podzolic A } \\
\text { horizon }\end{array}$ \\
\hline $\begin{array}{l}\text { Mount } \\
\text { Arrowsmith }\end{array}$ & \multicolumn{2}{|c|}{$13-255261$} & charcoal & 13000 & 640 & SUA 1959 & (Kiernan) & $\begin{array}{l}\text { palaeosol below } 1 \mathrm{~m} \\
\text { scree }\end{array}$ \\
\hline Scotts Peak Dam & \multicolumn{2}{|c|}{$11-421351$} & wood & $>33000$ & & Gak 5623 & Colhoun 1975 & $\begin{array}{l}\text { organic soil within } \\
\text { scree }\end{array}$ \\
\hline \multirow[t]{2}{*}{ Welcome River } & \multirow{2}{*}{\multicolumn{2}{|c|}{$86-111863$}} & charcoal & 30860 & $\begin{array}{r}+4100 \\
-2770\end{array}$ & Gak 5970 & Colhoun $1983 \mathrm{~b}$ & $\begin{array}{l}\text { palaeosol below } \\
\text { Holocene beach \& } \\
\text { dune }\end{array}$ \\
\hline & & & & \multicolumn{4}{|c|}{ MACQUARIE ISLAND } & \\
\hline Site Name & Lat & Long & Material & ${ }^{14} \mathrm{C}$ & S D & Lab No & Reference & Comment \\
\hline \multirow[t]{2}{*}{ Bauer Bay } & \multirow[t]{2}{*}{5030} & \multirow[t]{2}{*}{15853} & peat & 2165 & 80 & SUA 179 & $\begin{array}{l}\text { Colhoun \& } \\
\text { Goede } 1973\end{array}$ & min. age $9 \mathrm{~m}$ beach \\
\hline & & & $\begin{array}{l}\text { king } \\
\text { penguin } \\
\text { bones }\end{array}$ & 3980 & 140 & Gak 644 & $\begin{array}{l}\text { McEvey \& } \\
\text { Vestjens } 1974\end{array}$ & $7 \mathrm{~m}$ depth \\
\hline Finch Creek & 5435 & 15855 & $\begin{array}{l}\text { royal } \\
\text { penguin } \\
\text { bones }\end{array}$ & 6100 & 120 & Gak 643 & $\begin{array}{l}\text { McEvey \& } \\
\text { Vestjens } 1974\end{array}$ & $\begin{array}{l}\text { in } 6 \mathrm{~m} \text { terrace near } \\
\text { river mouth }\end{array}$ \\
\hline \multirow[t]{6}{*}{$\begin{array}{l}\text { Finch Creek, } \\
\text { Ridge }\end{array}$} & \multirow[t]{6}{*}{5434} & \multirow[t]{6}{*}{15855} & peat & 3780 & 140 & SUA 1460 & $\begin{array}{l}\text { Selkirk \& } \\
\text { Selkirk } 1983\end{array}$ & $1.27 \mathrm{~m}$ depth \\
\hline & & & sandy peat & 10275 & 230 & Beta 1386 & & $1.82 \mathrm{~m}$ \\
\hline & & & sandy peat & 5930 & 240 & SUA $1845 X$ & $\begin{array}{l}\text { Selkirk et al. } \\
1984\end{array}$ & $1.9 \mathrm{~m}$ \\
\hline & & & sandy peat & 5260 & 85 & Beta 7027 & $\begin{array}{l}\text { (Selkirk \& } \\
\text { Selkirk) }\end{array}$ & $1.9-2.0 \mathrm{~m}$ \\
\hline & & & humic acid & 5430 & 80 & Beta 7027B & & of Beta 7027 \\
\hline & & & sandy peat & 5630 & 80 & SUA 2126 & & $1.9-2.0 \mathrm{~m}$ \\
\hline \multirow[t]{3}{*}{ Gentoo Flat } & \multirow[t]{3}{*}{5432} & \multirow[t]{3}{*}{15853} & peat & modern & & Beta 6735 & (Dodson) & $0.05 \mathrm{~m}$ \\
\hline & & & peat & modern & & Beta 6736 & & $0.05-0.10 \mathrm{~m}$ \\
\hline & & & peat & modern & & Beta 6737 & & $0.25-0.28 \mathrm{~m}$ \\
\hline Green Gorge & 5438 & 15854 & peat & 2074 & 80 & SUA 178 & $\begin{array}{l}\text { Colhoun \& } \\
\text { Goede } 1973\end{array}$ & min. age $9 \mathrm{~m}$ beach \\
\hline \multirow[t]{2}{*}{$\begin{array}{l}\text { Green Gorge } \\
\text { Lake }\end{array}$} & \multirow[t]{2}{*}{5438} & 15854 & peat & 1570 & 140 & SUA 1463 & $\begin{array}{l}\text { Selkirk \& } \\
\text { Selkirk } 1983\end{array}$ & $1.2-1.4 \mathrm{~m}$ depth \\
\hline & & & peat & 7630 & 280 & SUA 1465 & & $2.2-2.3 \mathrm{~m}$ \\
\hline \multirow[t]{3}{*}{$\begin{array}{l}\text { Green Gorge } \\
\text { Ridge }\end{array}$} & \multirow[t]{3}{*}{5438} & 15854 & peat & 5140 & 140 & SUA 1462 & $\begin{array}{l}\text { Selkirk et al. } \\
1984\end{array}$ & $0.88 \mathrm{~m}$ depth \\
\hline & & & peat & 6900 & -800 & SUA 1461 & $\begin{array}{l}\text { Selkirk \& } \\
\text { Selkirk } 1983\end{array}$ & $1.38 \mathrm{~m}$ \\
\hline & & & peat & 7200 & 130 & SUA 2164 & $\begin{array}{l}\text { Selkirk et al. } \\
1984\end{array}$ & $\begin{array}{l}\text { repeat SUA } 1461 \\
\text { from adjacent profile }\end{array}$ \\
\hline \multirow{7}{*}{$\begin{array}{l}\text { Perseverance } \\
\text { Bluff }\end{array}$} & \multirow[t]{7}{*}{5430} & \multirow[t]{7}{*}{15856} & peat & 220 & 55 & Beta 8080 & (Dodson) & $0.15-0.2 \mathrm{~m}$ \\
\hline & & & peat & $<75$ & & Beta 8081 & & $0.2-0.25 \mathrm{~m}$ \\
\hline & & & peat & 300 & 55 & Beta 8082 & & $0.25-0.3 \mathrm{~m}$ \\
\hline & & & peat & 385 & 60 & Beta 8083 & & $0.3-0.35 \mathrm{~m}$ \\
\hline & & & peat & 625 & 60 & Beta 8084 & & $0.35-0.4 \mathrm{~m}$ \\
\hline & & & peat & 1245 & 80 & Beta 8085 & & $0.4-0.45 \mathrm{~m}$ \\
\hline & & & peat & 1685 & 70 & Beta 8086 & & $0.5-0.58 \mathrm{~m}$ \\
\hline
\end{tabular}




\begin{tabular}{|c|c|c|c|c|c|c|c|}
\hline \multirow{2}{*}{ Site Name } & \multicolumn{6}{|c|}{ MACQUARIE ISLAND } & \multirow[b]{2}{*}{ Comment } \\
\hline & Lat Long & Material & ${ }^{14} \mathrm{C}$ & S D & Lab No & Reference & \\
\hline \multirow[t]{3}{*}{ Scoble Lake } & $5431 \quad 15856$ & org. mud & 3140 & 100 & Wk 407 & Salas 1983 & $\begin{array}{l}0.78 \mathrm{~m} \text { depth at core } \\
\text { base near eastern } \\
\text { edge of lake }\end{array}$ \\
\hline & & org. mud & 7160 & 260 & Wk 405 & & $\begin{array}{l}\text { top of core from } \\
\text { centre of lake }\end{array}$ \\
\hline & & org. mud & 8700 & 220 & Wk 349 & & $\begin{array}{l}\text { base core at } 2 \mathrm{~m} \\
\text { depth }\end{array}$ \\
\hline \multirow[t]{4}{*}{ The Nuggets } & 543215857 & peat & 1700 & 110 & SUA 1468 & $\begin{array}{l}\text { Selkirk \& } \\
\text { Selkirk } 1982\end{array}$ & $\begin{array}{l}0.47-0.5 \mathrm{~m} \text { in cliff } \\
\text { section }\end{array}$ \\
\hline & & org. clay & 8560 & 200 & SUA 1467 & & $1.47-1.5 \mathrm{~m}$ \\
\hline & & diatomite & 8230 & 240 & SUA 1466 & & $3.39-3.41 \mathrm{~m}$ \\
\hline & & lake clay & 9400 & 220 & SUA 1894 & $\begin{array}{l}\text { Selkirk et al. } \\
1984\end{array}$ & $4.4 \mathrm{~m}$ \\
\hline \multirow[t]{8}{*}{ Wireless Hill } & 543015857 & sandy peat & 760 & 100 & SUA 1681 & $\begin{array}{l}\text { Selkirk et al. } \\
1983\end{array}$ & $1.0-1.05 \mathrm{~m}$ depth \\
\hline & & peat & 1515 & 75 & SUA 1238 & $\begin{array}{l}\text { Selkirk \& } \\
\text { Selkirk } 1983\end{array}$ & $1.25-1.35 \mathrm{~m}$ \\
\hline & & sandy peat & 1600 & 130 & SUA 1459 & $\begin{array}{l}\text { Selkirk et al. } \\
1983\end{array}$ & $2.06-2.1 \mathrm{~m}$ \\
\hline & & humic acid & 1300 & 90 & SUA $1459 \mathrm{HA}$ & & of SUA 1459 \\
\hline & & sandy peat & 3490 & 210 & SUA 1682 & & $3.13-3.15 \mathrm{~m}$ \\
\hline & & sandy peat & 5140 & 140 & Beta 1387 & & $3.58-3.60 \mathrm{~m}$ \\
\hline & & sandy peat & 4880 & 90 & SUA 1527 & & $3.84-3.94 \mathrm{~m}$ \\
\hline & & humic acid & 4610 & 100 & SUA $1527 \mathrm{HA}$ & & of SUA 1527 \\
\hline
\end{tabular}

Banks, M.R. Department of Geology, University of Tasmania

Chick, N.K. Department of Geography, University of Tasmania

Colhoun, E.A. Department of Geography, University of Tasmania

Derbyshire, E. Department of Geography

University of Leicester, England

Dodson, J. Department of Geography, University of New South Wales

Gill, E.D. $\quad 1 / 47$ Wattle Valley Road, Canterbury, Victoria

Goede, A. Department of Geography, University of Tasmania

Green, D. Department of Biogeography and Geomorphology, Research School of Pacific Studies, Australian National University, Canberra

Hannan, D. Division of Teacher Education, Tasmanian College of Advanced Education, Newnham

Hope, G.S. Department of Geography, School of General Studies, Australian National University, Canberra

Jackson, W.D. Department of Botany, University of Tasmania

Jones, R. Department of Prehistory, Research
School of Pacific Studies, Australian National University, Canberra

Kiernan, K.W. Department of Geography. University of Tasmania

Ladd, P.G. Department of Botany, School of General Studies, Australian National University, Canberra

Macphail, M.K. Esso House, 127 Kent Street, Sydney

Noble, M. Institute of Arctic and Alpine Studies, Boulder, Colorado, U.S.A.

Peterson, J.A. Department of Geography, Monash University

Podger, F. CSIRO, Stowell Avenue, Hobart

Ranson, D. Tasmanian National Parks and Wildlife Service, Sandy Bay

Sansom, P.W. Petrecon Pty. Ltd., Hobart

Selkirk, D.R. School of Biological Sciences, Sydney University

Selkirk, P.M. School of Biological Sciences, Macquarie University

Stephens, N. Department of Geography, University College of Wales, Swansea

Thomas, I. Department of Geography, University of Tasmania

van de Geer, G. Department of Geography, University of Tasmania 


\section{REFERENCES}

BANKS, M R COLHOUN, E A \& VAN DE GEER, G., 1976: Late Quaternary Palorchestes azael (Mammalia, Diprotodontidae) from northwestern Tasmania. Alcheringa 1: 159-166

BARENDSEN, G.W., DEEVEY, E.S.\& GRALENSKI, L.J., 1957: Yale Natural Radiocarbon Measurements 111. Science 126: 908-919.

CAINE, N. 1968: Carbon-14 dates from the Broad River Valley, Mt. Field National Park, Tasmania. Aust. J. Sci. 31 (3): 119

CAINE, N., 1978: Late Holocene instability on hillslopes in northeastern Tasmania. Search 9: 298.

CAINE, N., 1983: THE MOUNTAINS OF NORTHEASTERN TASMANIA. Balkema, Rotterdam.

COLHOUN, E.A., 1975: A Quaternary climatic curve for Tasmania. Conference paper at R. Met. Soc. Austr. Branch Conference on Climate and Climatic Change, Monash University, Dec., 1975, $40 \mathrm{pp}$.

COLHOUN, E.A., 1976: The glaciation of the lower Forth Valley, northwestern Tasmania, Aust. Geogr. Studies, 14(2): 83-102.

COLHOUN, E.A., $1977 \mathrm{a}$ : A sequence of Late Quaternary deposits at Pipe Clay Lagoon, southeastern Tasmania. Pap. Proc. R. Soc. Tasm. 111:29-39.

COLHOUN, E.A., 1977b. Late Quaternary fan gravels and slope deposits at Rocky Cape, northwestern Tasmania: their palaeoenvironmental significance, Pap. Proc. R. Soc. Tasm. 111: 13-28.

COLhoUN, E.A., 1977 c: The Remarkable Cave, southeastern Tasmania: its geomorphological development and environmental history. Pap. Proc. $R$. Soc. Tasm. 111: 29-39.

COLHOUN, E.A., 1979: QUATERNARY EXCURSION TO NORTHWEST AND WEST TASMANIA. FIELD GUIDE. 50 pp. Department of Geography, University of Tasmania.

COLHOUN, E.A., 1980: Quaternary fluviatile deposits from the Pieman Damsite, Western Tasmania. Proc. R. Soc. Lond. B207: 355-384.

COLHOUN, E.A., 1983a: Tasmanian sea levels in the last 15,000 years. In Hopley, D. (Ed.): AUSTRALIAN SEA LEVELS IN THE LAST 15,000 YEARS: A REVIEW. Monograph Series, Occasional Paper 3, Department of Geography, James Cook University: 54-58.

COLHOUN, E.A., 1983b: The Climate of Tasmania 32 \pm 5 KA. In PROCEEDINGS OF THE FIRST CLIMANZ CONFERENCE. Department of Biogeography and Geomorphology, Research School of Pacific Studies, Australian National University: $10-11$

COLHOUN, E.A., 1985: Glaciation of the West Coast Range, Tasmania. Quaternary Research 23(3) (in press).

COLHOUN, E.A. \& GOEDE, A., 1973: Fossil penguin bones, ${ }^{14} \mathrm{C}$ dates and the raised marine terrace of Macquarie Island: some comments. Search 4 (11-12): 499-501.

COLHOUN, E.A. and GOEDE, A., 1979: The Late Quaternary deposits of Blakes Opening and the
Middle Huon Valley, Tasmania. Phil. Trans. R. Soc Lond. 286 B: 371-395.

COLHOUN, E.A. \& MOON, A. 1984: Estuarine sediments at the Bowen Bridge on the Derwent River, southeastern Tasmania, Search 15 (7-8): $224-226$.

COLHOUN, E.A., VAN DE GEER, G. \& MOOK W.G., 1982: Stratigraphy, pollen analysis and palaeoclimatic interpretation of Pulbeena Swamp, northwestern Tasmania. Quaternary Research 18(1): 108-126.

COSGROVE, R.F., 1985: Eariy Holocene aboriginal occupation in northeastern Tasmania. Aust Archaeology (in press).

DAVIES, J.L., 1974: Geomorphology and Quaternary environments. In Williams, W.D. (Ed.): $B I O$ GEOGRAPHY AND ECOLOGY IN TAS$M A N I A$. Junk, The Hague: 17-27.

DERBYSHIRE, E., 1968: Two gelifluctates near Great Lake, central Tasmania. Aust. J. Sci. 31(4): 154-156.

FISH, G.J. \& YAXLEY, M.L., 1966: BEHIND THE SCENERY. Education Department, Tasmania.

FRANCEY, R.J., BAR BETTI, M., BIRD, T., BEARDSMORE, D., COUPLAND, W., DOLEZAL, J.E., FARQUHAR, G.D., FLYNN, R.G. FRASER, P.J., GIFFORD, R.M., GOODMAN, H.S., KUNDA, B., McPHAIL, S. NANSON, G., PEARMAN, G.I. RICHARDS, N.G., SHARKEY, T.D., TEMPLE, R.B. \& WEIR, B., 1984: Isotopes in tree rings. Division of Atmospheric Research Technical Paper No. 4, CSIRO, Australia.

GILL, E.D., 1956: Radiocarbon dating for glacial varves in Tasmania Aust. J. Sci. 19: 80.

GILL, E.D., 1968: Aboriginal bone implement from fossil bone bed, Tasmania. Rec. Q. Vict. Mus, Launceston 31: I-4.

GILLESPIE, R., 1977: Sydney University Natural Radiocarbon Measurements IV Radiocarbon, 19 $101-110$.

GOEDE, A., 1965: Geomorphology of the Buckland Basin. Pap. Proc. R. Soc. Tasm. 99: 133-154

GOEDE, A., 1973: Floodplain stratigraphy of the Tea Tree Rivulet, Buckland, eastern Tasmania. Austr. Geogr. Studies 11:28-39.

GOEDE, A. \& HARMON, R.S., 1983: Radiometric dating of Tasmanian speleothems - evidence of cave evolution and climatic change. J.Geol. Soc. Aust. 30: 89-100.

GOEDE, A.\& HITCHMAN, M.A., 1984: Late Quaternary climatic change - Evidence from a Tasmanian speleothem. In Vogel, J.C. (Ed.); LATE CAINOZOIC PALAEOCLIMATES OF THE SOUTHERN HEMISPHERE. Balkema, Rotterdam, 221-232.

GOEDE, A. \& MURRAY, P., 1979: Late Pleistocene bone deposits from a cave in the Florentine Valley, Tasmania. Pap. Proc. R. Soc. Tasm. 113 $39-52$. 
GRANT-TAYLOR, T.L.\& RAFTER, T.A., 1962: New Zealand radiocarbon age measurements: 5 . N.Z. J. Geol. Geophys. 5(2): 331-359.

GR ANT-TAYLOR, T.L. \& RAFTER, T.A., 1963: New Zealand natural radiocarbon measurements IV. Radiocarbon 5: 118-162.

GROOTES, P.M., 1978: Carbon-14 time scale extended comparison of chronologies. Science 200: 11-15.

KIGOSHI, K. \& KOBAYASHI, H., 1966: Gakushuin natural radiocarbon measurements V. Radiocarbon 8: 54-73.

KIGOSHI, K., AIZAWA, H. \& SUZUKI, N., 1969: Gakushuin natural radiocarbon measurements VI1. Radiocarbon 11 (2): 295-326.

L.ADD, P.G., 1981: The relevance of palynology to archaeology. The Artefact 6: 17-34.

MACPHAIL, M.K., 1979: Vegetation and climates in southern Tasmania since the Last Glaciation. Quaternary Research 11:306-341.

MACPHAIL, M.K., 1984: Small-scale dynamics in an Early Holocene wet sclerophyll forest in Tasmania. New Phytol. 96: 131-147.

MACPHAIL, M.K. \& COLHOUN, E.A., 1985: Late Last Glacial vegetation, climates and fire activity in southwest Tasmania. Search 16 (2): 43-45.

MACPHAIL, M.K.\& HOPE, G.S., 1985: Late Holocene mire development in montane southeastern Australia: a sensitive climatic indicator. Search $15(11-12): 344-349$.

MACPHAIL, M.K \& JACKSON, W.D. 1978: The Late Pleistocene and Holocene history of the Midlands of Tasmania. Proc. R. Soc. Vict. 90 (2): 287-300.

MACPHAIL, M.K. \& PETERSON, J.A., 1975: New deglaciation dates from Tasmania. Search 6: 127-130.

McEVEY, A.R. \& VESTJENS, W.J.M., 1974: Fossil penguin bones from Macquarie Island, Southern Ocean. Proc. R. Soc. Vict., 86 (2): I5I-174.

McPHAIL, S., BARBETTI, M., FRANCEY, R., BIRD, T. \& DOLEZAL, J., 1983: ${ }^{14} \mathrm{C}$ variations from Tasmanian trees - preliminary results. Radiocarbon 25 (3): 797-802.

MURRAY, P. \& GOEDE, A., 1977: Pleistocene vertebrate remains from a cave near Montagu, N,W. Tasmania Rec. Q. Vict. Mus. Launceston, 60.
PETERSON, J.A., 1968: Cirque morphology and Pleistocene ice formation conditions in southeastern Australia. Austr. Geogr. Studies 6: 67-83.

SALAS, M.R., 1983: Long-distance pollen transport over the southern Tasman Sea: evidence from Macquarie Island. N.Z.J. Bot. 21: 285-292.

SELKIRK, D.R.\& SELKIRK, P.M. 1983: Preliminary report on some peats from Macquarie lsland 7 +/-2KA. PROCEEDING OF THE FIRST CLIMANZ CONFERENCE. Department of Biogeography and Geomorphology. Research School of Pacific Studies, Australian National University: 115-117.

SELKIRK, D.R., SELKIRK, P.M., \& GRIFFIN, K., 1983: Palynological evidence for Holocene environmental change and uplift on Wireless Hill, Macquarie lsland. Proc. Linn. Soc., N.S.W. 107: $1-17$.

SELKIRK, P.M.\& SELKIRK, D.R., 1982: Late Quaternary mosses from Macquarie Island. J. HATTON BOTANICAL LABORATORY 52: 167-169.

SELKIRK, P.M. SELKIRK, D.R. \& BERGSTROM, D.M., 1984: Holocene vegetation history of Macquarie Island. The Tasmanian Naturalist $21-22$.

SIGLEO, W.R. \& COLHOUN, E.A., 1982: Terrestrial dunes, Man and the Late Quaternary environment in southern Tasmania. Palaeogeography, Palaeoclimatology, Palaeoecology. 39: 87-121.

STOCKTON, J., 1981: Radiocarbon dates for archaeological sites in Tasmania. Aust. Archaeology 12: 97-101.

THOM, B.G., BOWMAN, G.M., GILLESPIE, R. TEMPLE, R. \& BARBETTI, M., 1981: RADIOCARBON DATING OF HOLOCENE BEACHRIDGE SEOUENCES IN SOUTH-EAST AUSTRALIA. Monograph 11, Department of Geography, University of N.S.W. Royal Military College, Duntroon.

WASSON, R.J., 1977: Catchment processes and the evolution of alluvial fans in the lower Derwent Valley, Tasmania. Zeitschrift fur Geomorphologie NF 21(2): 147-168.

(accepted march 1, 1985) 